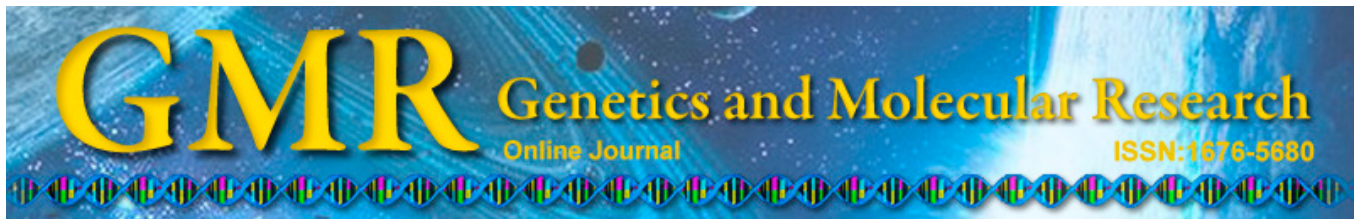

\title{
Allogeneic hematopoietic stem cell transplantation in children with aplastic anemia
}

H.-M. Xue, H.-G. Xu, K. Huang, H.-X. Guo, Y. Li, D.-H. Zhou, S.-L. Huang, J.-P. Fang and C. Chen

Department of Pediatrics, Sun Yat-Sen Memorial Hospital, Sun Yat-Sen University, Guangzhou, China

Corresponding author: C. Chen

E-mail: chunchendoc@126.com

Genet. Mol. Res. 14 (2): 5234-5245 (2015)

Received July 31, 2014

Accepted January 29, 2015

Published May 18, 2015

DOI http://dx.doi.org/10.4238/2015.May.18.15

ABSTRACT. The aim of this study was to prospectively investigate the efficacy and safety of fully matched allogeneic hematopoietic stem cell transplants in children with severe aplastic anemia in China. A total of twenty patients with severe aplastic anemia were enrolled in our study. Thirteen cases underwent transplantation with fully human leukocyte antigen (HLA)-matched, granulocyte-colony stimulating factor (G-CSF)-primed bone marrow and peripheral blood stem cells (PBSCs) from matching sibling donors. One patient received fully HLAmatched bone marrow from an unrelated donor. Six patients received fully HLA-matched G-CSF-primed PBSCs from unrelated donors. The conditioning regimen included fludarabine, cyclophosphamide, and rabbit anti-thymocyte globulin. Graft-versus-host disease prophylaxis was conducted with cyclosporin A and short-course methotrexate. The median follow-up duration was 3.08 years (range, 0.83-8.41years). The median time of neutrophil recovery $\left(>0.5 \times 10^{9} / \mathrm{L}\right)$ was 14 days (range, 10-20 days), and the median time of platelet recovery ( $>20 \mathrm{x}$ 
$10^{9} / \mathrm{L}$ ) was 19 days (range, 14-31 days). The survival rate at the cutoff point of follow-up was $95.0 \%$ (19/20). Initial engraftment rate was $95 \%$ $(19 / 20)$. Late graft failure (graft failures occurring 1 year or longer after transplantation) was observed in one patient. Only one patient developed Grade I acute graft-versus-host disease. Two cases suffered from EpsteinBarr virus (EBV)-associated post-transplant lymphoproliferative disorder and remitted after treatment with rituximab. One patient was diagnosed with hyperthyroidism 2.5 years after transplantation. Our study indicated that allogeneic hematopoietic stem cell transplantation is an effective and safe treatment for children with severe aplastic anemia in China.

Key words: Allogeneic hematopoietic stem cell transplantation; Pediatric; Acquired aplastic anemia

\section{INTRODUCTION}

Severe aplastic anemia (SAA) is a potentially fatal marrow failure syndrome characterized by hypocellular bone marrow and pancytopenia. The incidence rate varies widely, i.e., from two people per million in Western countries to four to six people per million in Asia (Young et al., 2006; Young and Kaufman, 2008). SAA risk factors in Asia include the high prevalence of viral hepatitis, as well as disparities in immunogenetic and unknown environmental factors among different ethnic groups.

Over the last decades, the overall prognosis for children with idiopathic SAA has improved significantly. The long-term disease-free survival rate is over $90 \%$ with human leukocyte antigen (HLA)-matched sibling donor allogeneic hematopoietic stem cell transplantation (AHSCT) (Samarasinghe et al., 2012). However, only $20-30 \%$ of patients have HLA-matched siblings. Thus, most patients with SAA receive initial treatments by immunosuppressive therapy (IST), and 10-35\% of patients relapse (Samarasinghe and Webb, 2012). Given the developments in HLA typing, effective dose-reduced conditioning regimens, and donor graft engineering, improved outcomes have been reported, even in children who have received transplants from well-matched unrelated donors (URDs) after failing IST. However, the outcome of allogeneic stem cell transplantation is hampered by the risk of graft rejection and graft-versus-host disease (GVHD) (Bacigalupo, 2012).

According to researchers in East Asia, the use of granulocyte-colony stimulating factor (G-CSF)-primed allogeneic bone marrow cells with G-CSF-mobilized peripheral blood stem cells (PBSCs) as the combined stem cell source for AHSCT results in rapid and stable engraftment without increasing the risk of acute and chronic GVHD during SAA treatment

(Sun et al., 2010). In the present study, we report findings on AHSCT cotransplantation for children with SAA in our hospital.

\section{MATERIAL AND METHODS}

\section{Patient and donor selection}

From August 1, 2005 to November 30, 2013, 118 patients were diagnosed with aplas- 
tic anemia in our unit. Among them, 98 patients received IST. Twenty patients underwent allotransplantation with a G-CSF-primed graft from HLA-related donors or URDs. Patients received immunotherapy or allogeneic stem cell transplantation with the following selection criteria: the family's economic status, and whether there was a suitable donor. All patients enrolled in this study were diagnosed with SAA according to the Camitta criteria. Bone marrow biopsy and cytogenic analysis were applied to exclude hypoplastic myelodysplastic syndrome and Fanconi anemia. This study was conducted in accordance with the Declaration of Helsinki and with approval from the Ethics Committee of Sun Yat-Sen Memorial Hospital. Written informed consent was obtained from all participants.

Low-resolution genotyping was performed on eight patients (Cases 1 to 8 ) and their sibling donors; HLA-A, HLA-B, and HLA-DR were fully matched. High-resolution genotyping was performed on five patients (Cases 9 to 13) and their sibling donors; HLA-A, HLA-B, HLA-Bw, HLA-DRB1, and HLA-DRw were fully matched. High-resolution genotyping was also performed on seven patients (Cases 14 to 20) and their URDs; HLA-A, HLA-B, HLA-Bw, HLA-DRB1, and HLA-DRw were fully matched except for the HLA-DRw antigen of Case 15. All the seven matched URDs (MUDs) came from the China Marrow Donor Program (CMDP). No patient or donor was positive for cytomegalovirus (CMV) or hepatitis B virus antigen or antibody. At the time of transplantation, 15 patients had no evidence of acute infection. However, Case 10 had severe fungal pneumonia, and Case 17 had severe Enterococcus faecalis septicemia with high fever lasting for more than 1 month. Although the patients were treated with powerful antibacterial and antifungal agents, the infections were not controlled until engraftment.

\section{Transplantation}

Thirteen cases underwent transplantation with fully HLA-matched G-CSF-primed bone marrow and PBSCs from a matched sibling donor. All thirteen donors were given 5-8 $\mathrm{mg} \cdot \mathrm{kg}^{-1} \cdot$ day $^{-1}$ subcutaneous or intravenous G-CSF (Filgrastim ${ }^{\circledR}$ or Lenograstim ${ }^{\circledR}$ ) for 5-7 days. On the fifth day, cytocentrifugation (Baxter CS 3000 Plus, Baxter, Deerfield, IL, USA) was used to collect PBSCs, and bone marrow was collected from the posterior iliac crest under local or epidural anesthesia. Red blood cells in the bone marrow were precipitated with hydroxyethyl starch for major donor-recipient $\mathrm{ABO}$ mismatch cases. One patient received fully HLA-matched bone marrow from an URD. Six patients received G-CSF-primed PBSCs from URDs. The cell dose infusion and transplantation data are shown in Table 1.

\section{Conditioning regimen}

A combination of fludarabine (FLU), cyclophosphamide (CTX), and rabbit anti-thymocyte globulin (ATG) was administered to 19 cases. One case received total body irradiation (TBI), as well as FLU and CTX. FLU was administered at atotal dose of $125 \mathrm{mg} / \mathrm{m}^{2}(\mathrm{~N}=5)$ or $150 \mathrm{mg} / \mathrm{m}^{2}(\mathrm{~N}=15)$ for 20 patients and was administered separately on days 6,5 , and 4 before transplantation. CTX was administered at a total dose of $120 \mathrm{mg} / \mathrm{kg}$ for 9 patients and $200 \mathrm{mg} /$ $\mathrm{kg}$ for 11 patients, and was administered separately on days 3 and 2 before transplantation. ATG was administered at a total dose of $22.5 \mathrm{mg} / \mathrm{kg}$ for 14 patients, $12.5 \mathrm{mg} / \mathrm{kg}$ for 4 patients, and $7.5 \mathrm{mg} / \mathrm{kg}$ for 1 patient, and was administered separately on days 3,2 , and 1 before trans- 
plantation. TBI was performed in one session (dose, $3 \mathrm{~Gy}$ ) for 1 case. The reason for TBI was that there was no medication towards ATG during the transplantation. The reason for the different dosages of FLU and ATG was that the children with the lower dosage had received reinforced therapy 6 months earlier, and the donor and recipient were siblings, or even twins, so it was expected that the lower dosage would reduce side effects.

\section{GVHD prophylaxis}

Cyclosporin A (CSA) and methotrexate were given to all patients for GVHD prophylaxis. CSA was given as a continuous intravenous dose of $3 \mathrm{mg} \cdot \mathrm{kg}^{-1} \cdot \mathrm{day}^{-1} 1$ day before transplantation until hospital discharge and then orally at $5-8 \mathrm{mg} \cdot \mathrm{kg}^{-1} \cdot \mathrm{day}^{-1}$. The blood concentration of CSA was regularly monitored and maintained at 200-400 $\mu \mathrm{g} / \mathrm{L}$. Methotrexate was given at $10 \mathrm{mg} / \mathrm{m}^{2}$ on days 3,6 , and 11 after transplantation. Surveillance for acute GVHD consisted of routine blood testing, body temperature monitoring, skin checks, abdominal examination, and liver function tests. The expected manifestations of GVHD were fever, rash, liver dysfunction with jaundice, and diarrhea.

\section{Infection prophylaxis}

Conventional anti-virus prophylaxis was performed prior to transplantation, beginning with ganciclovir at $10 \mathrm{mg} \cdot \mathrm{kg}^{-1} \cdot \mathrm{day}^{-1}$ for 7 days. The dosage was changed to 5 $\mathrm{mg} \cdot \mathrm{kg}^{-1} \cdot \mathrm{day}^{-1}$ for 7 days until the day before transplantation. Patients were also nursed during this period in laminar airflow rooms and were fed a low-microbial diet. Sulfamethoxazole-trimethoprim was administered just before transplantation, and oral itraconazole was given as antifungal prophylaxis. Empiric antibiotics were given for bacterial or fungal infection as clinically indicated. Regular CMV-Ag and CMV-PP65 testing, CMV-DNA as well as Epstein-Barr virus (EBV)-DNA polymerase chain reactions, were performed after transplantation. The $\mathrm{CMV}$ polymerase chain reaction conditions were as follows: $95^{\circ} \mathrm{C}$ pre-denaturation for $2 \mathrm{~min}, 95^{\circ} \mathrm{C}$ denaturation for $45 \mathrm{~s}, 57^{\circ} \mathrm{C}$ annealing for $45 \mathrm{~s}$ for 40 cycles, and $37^{\circ} \mathrm{C}$ for $10 \mathrm{~s}$.

\section{Hemorrhagic cystitis}

Hydration therapy at a dosage of $3000-3500 \mathrm{~mL} \cdot \mathrm{m}^{-2} \cdot$ day $^{-1}$ was initiated $6 \mathrm{~h}$ before the conditioning regimen. A diuretic was administered to achieve $3 \mathrm{~mL} \cdot \mathrm{kg}^{-1} \cdot \mathrm{h}^{-1}$ urinary output. Sodium 2-mercaptoethane sulfonate $\left(200-400 \mathrm{mg} \cdot \mathrm{m}^{-2} \cdot\right.$ session $\left.^{-1}\right)$ and CTX was given intravenously for $24 \mathrm{~h}$ prior to initiating the conditioning regimen.

\section{Detection of successful engraftment and clinical follow-up}

In patients with donor/recipient sex-mismatched grafts, donor chimerism was evaluated based on the results of two-color fluorescent in situ hybridization (FISH) sex chromosome analysis. In patients with sex-matched grafts, engraftment success was examined through ABO blood group detection before 2009. From 2010, variable number tandem repeat 
polymorphisms were used to monitor donor chimerism for sex-matched grafts.

This study was conducted from August 2005 to November 2013 with a median duration of follow-up of 3.08 years (range, 0.83-8.41years; Table 1).

\section{RESULTS}

\section{Hematopoietic reconstitution, successful engraftment, and GVHD}

Successful transplantation was documented in all patients except for Case 4. Repeat FISH analysis identified unsuccessful engraftment in Case 4 at the first and third months after transplantation. However, results of the repeat peripheral blood test were normal, thus indicating unsuccessful engraftment with the possibility of the simultaneous recovery of autologous hematopoiesis for the Case 4 patient, who was 3.25 years old at the time of transplantation and was diagnosed with SAA not more than 2 months prior to transplantation.

The median time to neutrophil recovery $\left(>0.5 \times 10^{9} / \mathrm{L}\right)$ was 14 days (range, 10-20 days), whereas the mean time to platelet recovery $\left(>20 \times 10^{9} / \mathrm{L}\right.$ ) was 19 days (range, 14-31 days) (Table 1).

Normal bone marrow morphology was observed 1 month after transplantation in 14 cases. Two months after transplantation, normal bone marrow morphology was found in three cases. Although the bone marrow examination of Case 2 was normal at the first and second months after transplantation, erythroid cellularity decreased at the sixth month and hypoplasia of both erythroid cellularity and granulocyte cellularity was observed at the 17th month. Recurrent SAA was diagnosed, and the child died of respiratory failure.

\section{EBV and post-transplant lymphoproliferative disorder (PTLD)}

In our study, seven patients received IST with ATG 6-15 months before allotransplantation. By contrast, thirteen patients did not receive IST with ATG (Table 2). Prior to ATG treatment, the quantitative detection of blood EBV-DNA was conducted in six patients (Cases 7, 8, 15,16, 18, and 19) before and after transplantation. Four cases (Cases 7, 16,18 , and 19) had significantly high EBV-DNA load pre-transplantation $\left(9.77 \times 10^{7}, 2.76\right.$ x $10^{3}, 5.68 \times 10^{5}$, and $3.24 \times 10^{3}$ copies $/ \mathrm{mL}$ ) without any clinical symptoms. After transplantation, all six patients exhibited significantly high values of EBV-DNA load ( $8.73 \mathrm{x}$ $10^{5}$ to $8.62 \times 10^{8}$ copies $/ \mathrm{mL}$ ). Intravenous immunoglobulin and intravenous ganciclovir were started. The EBV-DNA copy numbers of four patients decreased gradually and became undetectable at 30,52,61, and 55 days post-transplantation. Case 16, who received ATG 15 months before allotransplantation, showed a continuously elevated EBV load (peak, $8.62 \times 10^{8}$ copies $/ \mathrm{mL}$ ), and suffered from PTLD at 24 days post-transplantation. The biopsy of a cervical lymph node revealed effacement of normal lymph node architecture by diffuse atypical/polyclonal middle or large immunoblastic cell hyperplasia. Immunophenotyping showed a mixture of $\mathrm{T}$ and $\mathrm{B}$ cells that expressed intracytoplasmic $\operatorname{IgH}$ clonal rearrangement. In situ hybridization confirmed positive EBV untranslated RNA that was consistent with EBV-associated PTLD (EBV-PTLD). 
Effective treatment for severe aplastic anemia

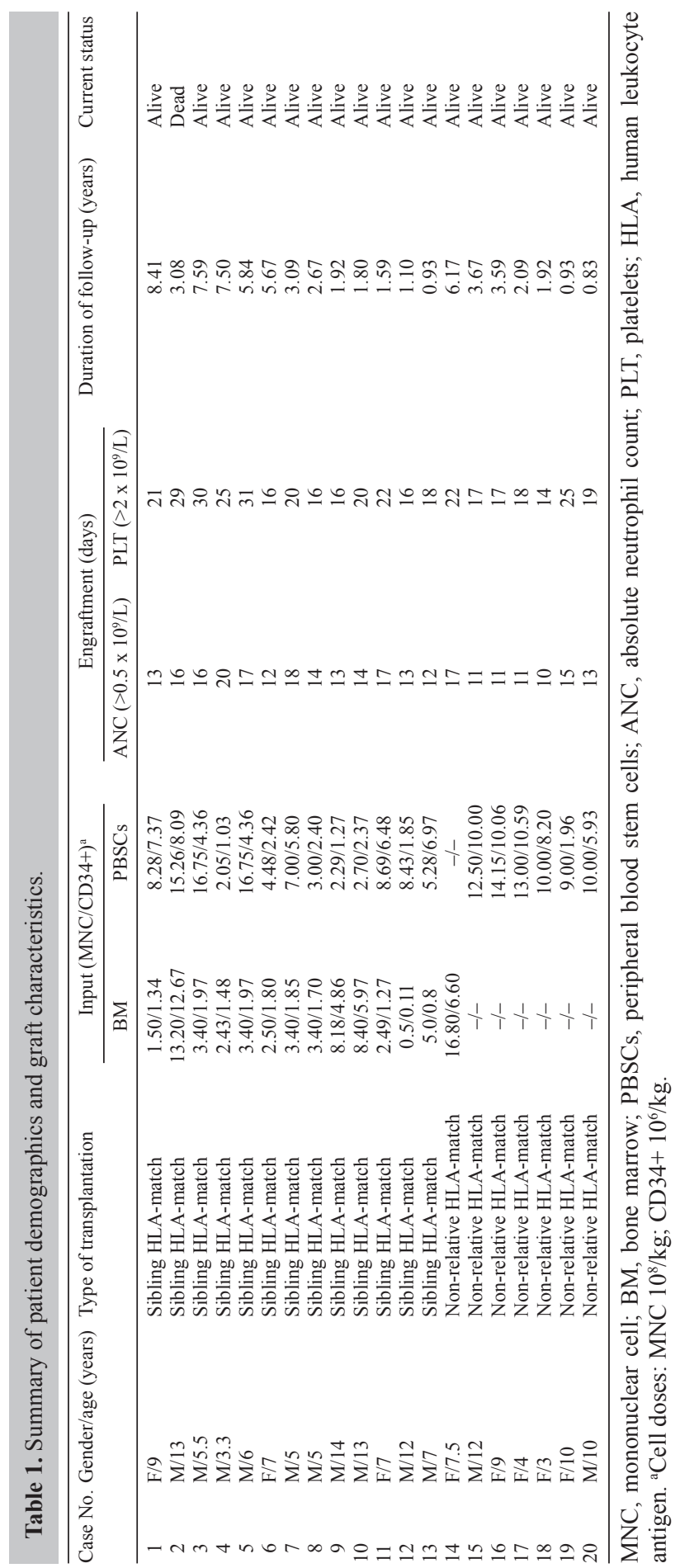


IST (i.e., CSA) was discontinued immediately, whereas rituximab and intravenous methylprednisolone were implemented. Rituximab was administered at $375 \mathrm{mg} / \mathrm{m}^{2}$ per week for 4 consecutive weeks, and a rapid response was noted. Two days after the first dose of rituximab was given, the fever of Case 16 decreased gradually and the lymph nodes began to shrink; after the second week, the lymph nodes were impalpable. The EBV load was also negative after the last dose of rituximab had been administered. During the treatment course of EBV-PTLD, FISH analysis showed successful engraftment, and bone marrow and peripheral blood tests both showed normal results. Up to the cutoff day, 12 months after transplantation, Case 16 remained in remission from both SAA and EBV-PTLD. The other case (Case 18), who received ATG 9 months before allo-transplantation, showed a continuously elevated EBV load (peak, $3.2 \times 10^{6} \mathrm{copies} / \mathrm{mL}$ ), and suffered from post-transplant lymphoproliferative disorder (PTLD) at 42 days post-transplantation. The biopsy of a cervical lymph node revealed effacement of normal lymph node architecture by diffuse atypical/polyclonal immunoblastic cell hyperplasia. Immunophenotyping showed B cells. In situ hybridization confirmed positive Epstein-Barr encoded RNAs1 that were consistent with EBV-associated PTLD (EBV-PTLD). IST (i.e., CSA) was discontinued immediately, whereas rituximab were implemented. After the second dose of rituximab was given, all the clinical manifestations disappeared and the EBV load turned to negative. Up to the cutoff day, 1.92 yrs after transplantation, Case 18 remained in remission from both SAA and EBV-PTLD.

Blood EBV loads were monitored in only nine patients who had not undergone ATG treatment before transplantation (Table 2). No positive results were found before transplantation. Only one child (Case 9) had increased EBV-DNA copy number $\left(6.37 \times 10^{5}\right.$ copies $\left./ \mathrm{mL}\right)$ after transplantation. EBV-DNA copy numbers were normal from 33 days post-transplantation following therapy with intravenous immunoglobulin and intravenous ganciclovir.

\section{Other transplantation-related complications}

Transplantation went smoothly in all patients, i.e., no patient experienced hemorrhagic cystitis or hepatic veno-occlusive disease. Only Case 15 (Table 1), who received PBSCs from a URD with an HLA-DRw antigen mismatch, developed Grade I acute GVHD on the skin, but it was easily controlled by prednisone. Two patients (Cases 2 and 3) developed Escherichia coli on the ninth and second days post-transplantation. The patients recovered after piperacillin was administered. The other patients had low-grade fevers (range, $37.5-38.5^{\circ} \mathrm{C}$ ) in the interval between transplantation and normal hemogram development. Those patients recovered after antibiotic and supportive therapies were implemented. Two patients (Cases 10 and 17) had severe and uncontrolled infections before transplantation. The infections were controlled as soon as the white blood cell count was $>1.0 \times 10^{9} / \mathrm{L}$ after transplantation.

\section{Follow-up}

The cutoff date for follow-up was November 15,2013. None of the patients exhibited either acute or chronic GVHD during this time. Among the 20 patients, 19 were alive and 18 were disease-free at the cutoff point. Oral CSA therapy was continued for three patients (Cases 13, 19, and 20) at the end of the follow-up. Continued complete engraftment was documented in 19 cases at the most recent follow-up except for one child (Case 4). Recurrent aplastic anemia was diagnosed 17 months after transplantation in Case 2, in whom white blood cell, red blood cell, and platelet counts decreased significantly. 
Effective treatment for severe aplastic anemia

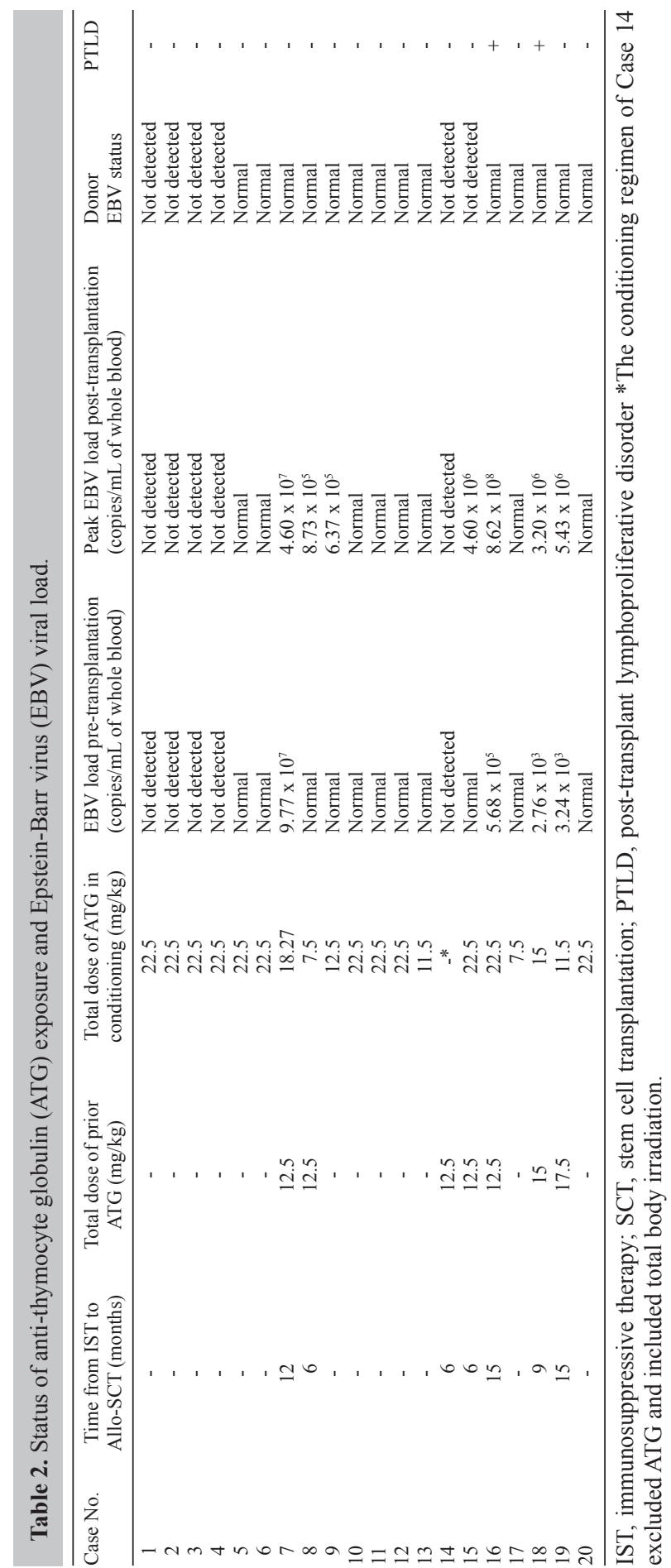


FISH sex chromosome detection indicated transplant rejection. Bone marrow examination indicated reduced erythroid cellularity and decreased granulocyte cellularity. Persistent hyperpyrexia, repeated epistaxis, and multiple subcutaneous abscesses also developed. Meropenem, imipenem, vancomycin, voriconazole, amphotericin B, acyclovir, and supportive therapy were provided. Case 2 died 28 months after transplantation because of respiratory failure. Case 4 failed engraftment because of autologous hematopoiesis recovery. CSA was given continuously. Peripheral blood tests and bone marrow aspiration were conducted for approximately 6 months with a normal value. However, SAA was recurrent in the case at the seventh month. The parents of Case 4 refused a second transplantation and ATG treatment. Consequently, Case 4 was given CSA combined with Chinese medicine (compound Zaofan pill, because practitioners of Chinese medicine thought that this medicine would warm the kidneys and strengthen the marrow, supplement Qi and nourish Yin, regenerate the blood and stop the bleeding, and thereby treat the aplastic anemia). To date, the patient needs a red blood cell transfusion every 2 months and a platelet transfusion every month. Approximately 2.5 years after transplantation, Case 5 presented nervousness, ocular proptosis, and emaciation. Hyperthyroidism was diagnosed owing to elevated serum FT4 and FT3 levels, as well as undetectable thyroid-stimulating hormone. Case 5 was treated with thiamazole. The symptom was controlled rapidly, i.e., FT3, FT4, and thyroid-stimulating hormone became normal after 2 months of treatment. Thiamazole was discontinued after 6 months. To date, the patient has been in hematologic remission for 5 years and 22 months with normal thyroid function.

\section{DISCUSSION}

The supply of marrow from young sibling donors is inadequate for transplantation, and one of the most important problems facing sibling bone marrow transplantation is the acquisition of sufficient stem cells. Following our previous encouraging report on bone marrow transplantation combined with PBSC for children with SAA (Chun et al., 2011), we continued this prospective trial by recruiting more patients and extending the follow-up time. To the best of our knowledge, this report on hematopoietic stem cell transplantation (HCT) was the first to use both G-CSF-mobilized PBSCs and bone marrow stem cells for children with SAA in China. Bone marrow transplantation combined with PBSCs could improve the number of nucleated cells from PBSCs, thus achieving rapid engraftment without increasing the risk of GVHD. Other studies have reported that transplantations can achieve successful engraftment by using dual-source stem cells primed with G-CSF for adults with SAA; only a very few patients had slight GVHD. In the current study, we expanded the number of subjects from 5 children to 13 patients, and the result was encouraging. The initial engraftment rate was $92.31 \%$ $(12 / 13)$. Late graft failure (graft failures occurring 1 year or longer after transplantation) was observed in one patient, and only one patient developed Grade I acute GVHD that was limited to the skin. The presumed mechanism was that the G-CSF-primed mixture of PBSCs and bone marrow mesenchymal stem cells might not only increase the numbers of stem cells and mesenchymal stem cells but also change the function of T-cells and other cells, such as natural killer cells, which contribute to engraftment and cause a relatively low GVHD rate (WeberMzell et al., 2007; Sun et al., 2010; Wang et al., 2012). These studies indicated that the dual collection of stem cells was a safe and effective way to improve the number of nucleated cells. The dual collection of stem cells also served an important function in successful engraftment without severe GVHD for children with SAA. We hypothesized that dual collection could be 
applied to all sibling donors to improve the number of nucleated cells from PBSCs and promote engraftment without severe GVHD for children with SAA.

The combination of ATG and CTX at a total dose of $200 \mathrm{mg} / \mathrm{kg}$ is widely used as the standard conditioning regimen for SAA (Hutspardol et al., 2013). FLU is an effective immunosuppressant and may possibly play a role in reducing both the incidence and severity of GVHD transplantation therapy (Anderlini et al., 2011; Tolar et al., 2012). The toxicity caused by CTX remains a significant challenge in treating children with SAA (Buchbinder et al., 2012). Recent studies indicate that CTX at a dose as low as $100 \mathrm{mg} / \mathrm{kg}$ in combination with FLU can achieve similar overall levels of complications and survival rates with significant low-incidence regimen-associated toxicities (Kim et al., 2012; Chung et al., 2013; Maury et al., 2013). The combination of FLU, ATG, and CTX (120 mg/m² for nine patients and $200 \mathrm{mg} /$ $\mathrm{m}^{2}$ for the remaining eleven patients) was used as the conditioning regimen in our study. The survival rate at the cutoff point was $94.11 \%$ with a median follow-up of 2.75 years in patients receiving HCT from either sibling or non-relative donors. Only one patient developed Grade I acute GVHD. The initial engraftment rate was $95 \%$, and late graft failure (graft failures occurring one year or longer after transplantation) was identified in one patient without significant toxicity caused by CTX. Although the sample size was inadequate, our encouraging results indicated that the low dose of CTX for fully matched (high-resolution) transplantation from a URD was suitable.

Discussions should focus on the implications of the fact that seven patients successfully underwent matched URD (MUD)-HCT, which was carried out because of the lack of matched sibling donors (MSD). These cases were successful to their last follow-up visit 3.09 years after transplantation. The key step towards a successful HCT is to obtain hematopoietic stem cells (HSCs) from a matched donor or matched sibling donor. Considering that the "family planning" policy has been implemented for more than three decades in China, most families only have one child. Thus, finding an available sibling donor in China is impossible. MUDs are usually the best choice for an alternative. In our study, the success of five cases that used HSC from MUDs was significant. Our success in MUD-HCT might be attributed to the short interval between diagnosis and HCT, as well as to the young age of the recipients. A multicenter retrospective study from the United Kingdom showed that the 5-year estimated failure-free survival (FFS) following IST was $13.3 \%$ for children with SAA. The 5-year FFS of children who received a 10-antigen MUD-HCT was 95.01\% (Peinemann et al., 2009). Other researchers have confirmed that transplants from MUDs are equivalent to transplants from MSDs, particularly in overall survival and FFS (Yagasaki et al., 2010; Samarasinghe et al., 2012; Chen et al., 2013). Our success may be attributed to the short interval between diagnosis and $\operatorname{HCT}(3,6,9,12,12,15$, and 15 months), the young age of the recipients $(3,4,7.5,9$, 10,10, and 12 years), and effective conditioning regimens, including effective T-cell depletion by fludarabine and ATG. Therefore, HCT might be considered if a MUD is available for newly diagnosed children with SAA.

Predictive risk factors for PTLD remain undetermined in allo-HCT. Buyck et al. (2009) reported that the overall incidence of EBV-PTLD in patients with acquired aplastic anemia receiving allotransplants was $6.3 \%$, and prior use of ATG strongly influenced the development of PTLD; in particular, those receiving multiple prior courses of ATG were at the highest risk. Presumably the reason is that T-cell dysfunction caused by multiple courses of ATG might create a unique environment for EBV replication. Such a pattern was also seen in our study, in which $60 \%$ of the patients who had previously received IST with ATG before al- 
lotransplantation had an abnormal EBV load pre-transplantation, compared with no EBV load in those not exposed to ATG before transplantation; the result was $5 / 5$ vs $1 / 7$ after transplantation. Two children suffered from EBV-PTLD had exposed to ATG in 15 and 9 months before HSCT. In our country, rabbit ATG is the only source of ATG. A recent study from the National Institutes of Health suggests that the use of rabbit ATG is associated with a higher EBV load compared with equine ATG, and therefore with the increased risk of EBV-PTLD after allo-HCT.

Our study has a number of limitations. The first is the small sample size. Owing to the encouraging outcomes from the previous study, the number of SAA children in our study increased nearly 3 times from 6 to 20, but was still comparatively small. The second limitation is grouping. In this study, HSC was obtained from either bone marrow or bone marrow combined with PBSCs. In subsequent studies, patients should be randomized into two groups. The third limitation is the relatively short follow-up time after transplantation. Fourth is that our study is not a random controlled trial. In subsequent studies, an IST group should be set up as a control to compare the outcomes of HCT and IST. However, our series of studies, including the previous study, have provided the first prospective reports on the outcomes of fully matched AHSCT in children with SAA in China.

Despite the limitations, our study provides valuable information for the treatment of SAA in children. To improve the outcomes of MUD-HCT, an effective conditioning regimen and prophylaxis for GVHD must be established. Our future objectives include the expansion of the donor registry, which is a service that is increasingly being accepted by Chinese people.

In summary, 20 children with SAA who underwent AHSCT with a median follow-up of 3.08 years achieved a 95\% survival rate with FLU-CTX-ATG as the conditioning regimen. The use of dual-source stem cells in this study contributed to the low GVHD-related incidence and mortality.

\section{Conflicts of interest}

The authors declare no conflict of interest.

\section{ACKNOWLEDGMENTS}

Research supported by a National Nature Scientific Grant of China (\#30772367), a Nature Scientific Grant of Guangdong Province (\#S2011010002648), and an International Cooperation Grant of Guangdong Province (\#2011B050400023).

\section{REFERENCES}

Anderlini P, Acholonu SA, Okoroji GJ, Bassett RE, et al. (2011). Fludarabine, cyclophosphamide, and antithymocyte globulin for matched related and unrelated allogeneic stem cell transplant in severe aplastic anemia. Leuk. Lymphoma 52: $137-141$.

Bacigalupo A (2012). Matched and mismatched unrelated donor transplantation: is the outcome the same as for matched sibling donor transplantation? Hematology Am. Soc. Hematol. Educ. Program 2012: 223-229.

Buchbinder D, Nugent DJ, Brazauskas R, Wang Z, et al. (2012). Late effects in hematopoietic cell transplant recipients with acquired severe aplastic anemia: a report from the late effects working committee of the center for international blood and marrow transplant research. Biol. Blood Marrow Transplant. 18: 1776-1784.

Buyck HC, Ball S, Junagade P, Marsh J, et al. (2009). Prior immunosuppressive therapy with antithymocyte globulin increases the risk of EBV-related lymphoproliferative disorder following allo-SCT for acquired aplastic anaemia. Bone Marrow Transplant. 43: 813-816. 
Chen J, Lee V, Luo CJ, Chiang AK, et al. (2013). Allogeneic stem cell transplantation for children with acquired severe aplastic anaemia: a retrospective study by the Viva-Asia Blood and Marrow Transplantation Group. Br. J. Haematol. 162: 383-391.

Chun C, Fang JP, Xue HM, Zhou DH, et al. (2011). Allogeneic hematopoietic stem cell transplantation for childhood aplastic anemia: Prospective trial in China. Transfus. Apher. Sci. 44: 41-47.

Chung NG, Lee JW, Jang PS, Jeong DC, et al. (2013). Reduced dose cyclophosphamide, fludarabine and antithymocyte globulin for sibling and unrelated transplant of children with severe and very severe aplastic anemia. Pediatr. Transplant. 17: 387-393.

Hutspardol S, Sirachainan N, Anurathapan U, Pakakasama S, et al. (2013). Allogeneic hematopoietic stem cell transplantation for children with severe aplastic anemia. J. Med. Assoc. Thai. 96: S18-S24.

Kim H, Lee JH, Joo YD, Bae SH, et al. (2012). A randomized comparison of cyclophosphamide $v s$ reduced dose cyclophosphamide plus fludarabine for allogeneic hematopoietic cell transplantation in patients with aplastic anemia and hypoplastic myelodysplastic syndrome. Ann. Hematol. 91: 1459-1469.

Maury S, Balère-Appert ML, Pollichieni S, Oneto R, et al. (2013). Outcome of patients activating an unrelated donor search for severe acquired aplastic anemia. Am. J. Hematol. 88: 868-873.

Peinemann F, Grouven U, Kröger N, Pittler M, et al. (2009). Unrelated donor stem cell transplantation in acquired severe aplastic anemia: a systematic review. Haematologica 94: 1732-1742.

Samarasinghe S and Webb DK (2012). How I manage aplastic anaemia in children. Br. J. Haematol. 157: 26-40.

Samarasinghe S, Steward C, Hiwarkar P, Saif MA, et al. (2012). Excellent outcome of matched unrelated donor transplantation in paediatric aplastic anaemia following failure with immunosuppressive therapy: a United Kingdom multicentre retrospective experience. Br. J. Haematol. 157: 339-346.

Sun ZM, Liu HL, Geng LQ, Wang XB, et al. (2010). HLA-matched sibling transplantation with G-CSF mobilized PBSCs and BM decreases GVHD in adult patients with severe aplastic anemia. J. Hematol. Oncol. 3: 51.

Tolar J, Deeg HJ, Arai S, Horwitz M, et al. (2012). Fludarabine-based conditioning for marrow transplantation from unrelated donors in severe aplastic anemia: early results of a cyclophosphamide dose deescalation study show life-threatening adverse events at predefined cyclophosphamide dose levels. Biol. Blood Marrow Transplant. 18: 1007-1011.

Wang H, Yan H, Wang Z, Zhu L, et al. (2012). Cotransplantation of allogeneic mesenchymal and hematopoietic stem cells in children with aplastic anemia. Pediatrics 129: e1612-e1615.

Weber-Mzell D, Urban C, Benesch M, Rojacher T, et al. (2007). Durable remission following a third allogeneic stem cell transplantation in a patient with repeatedly relapsing SAA. The importance of stroma cells for sustained engraftment. Pediatr. Transplant. 11: 332-335.

Yagasaki H, Takahashi Y, Hama A, Kudo K, et al. (2010) Comparison of matched-sibling donor BMT and unrelated donor BMT in children and adolescent with acquired severe aplastic anemia. Bone Marrow Transplant. 45: 1508-1513.

Young NS and Kaufman DW (2008). The epidemiology of acquired aplastic anemia. Haematologica 93: 489-492.

Young NS, Calado RT and Scheinberg P (2006). Current concepts in the pathophysiology and treatment of aplastic anemia. Blood 108: 2509-2519. 